\title{
Aplicação do Algoritmo de Cuthill-McKee em Matrizes de Hodge para o Método da Esparsificação Recursiva ${ }^{\dagger}$
}

\author{
J.L.P. LUIZ ${ }^{1 *}$, B.F.C. SILVA ${ }^{1}$, G.M.C. MAGALHAES ${ }^{1}$, A.S. MOURA ${ }^{2}$, \\ R.R. SALDANHA ${ }^{3}$ e E.J. SILVA ${ }^{3}$
}

Recebido em 29 novembro, 2013 / Aceito em 28 maio, 2015

\begin{abstract}
RESUMO. A solução de sistemas lineares esparsos de alta ordem está inserido em vários ramos da ciência, como por exemplo a engenharia. Por conseguinte, tem havido um grande esforço para resolver ou apresentar soluções aproximadas de tais sistemas de forma eficiente. Neste trabalho combina-se o método da esparsificação recursiva com o algoritmo de Cuthill-McKee para obter uma aproximação esparsa para a inversa de uma classe de matrizes esparsas denominadas matrizes de Hodge.
\end{abstract}

Palavras-chave: esparsificação recursiva, matriz de Hodge, algoritmo de Cuthill-McKee.

\section{INTRODUÇÃO}

A solução de sistemas esparsos de alta ordem está inserida em vários ramos da ciência, como por exemplo, a engenharia. Por conseguinte, tem havido um grande esforço para resolver ou apresentar soluções aproximadas de tais sistemas de forma eficiente.

Quando se trata de problemas de propagação de ondas eletromagnéticas utilizando a teoria de formas diferenciais [1]-[6], tem-se que as equações de Maxwell em sua forma semi-discreta se comportam como um sistema linear esparso de alta ordem. A solução de problemas desse tipo consiste na resolução de um sistema linear esparso definido pela inserção das relações constitutivas através das matrizes de Hodge em cada passo de tempo [5].

\footnotetext{
$\dagger$ Trabalho apresentado no Congresso de Matemática Aplicada e Computacional-Nordeste, em novembro de 2012, NatalRN.

*Autor correspondente: José Lucas Pereira Luiz

${ }^{1}$ Departamento de Ciências Exatas, DCEX, UFVJM - Universidade Federal dos Vales do Jequitinhonha e Mucuri, 39803-853 Teófilo Otoni, MG, Brasil.

E-mails: lucasvt09@hotmail.com; brunobfcs@ymail.com; gaublis@hotmail.com

2 Departamento de Economia, UFJF - Universidade Federal de Juiz de Fora, Av. Dr. Raimundo Monteiro Rezende, 330 , Centro, 35010-177 Governador Valadares, MG, Brasil. E-mail: alex.moura@ufjf.edu.br

${ }^{3}$ Departamento de Engenharia Elétrica, UFMG - Universidade Federal de Minas Gerais, Belo Horizonte, MG, Brasil. E-mails: rodney@cpdee.ufmg.br; elson@cpdee.ufmg.br
} 
Conforme [5], um FETD ${ }^{4}$ incondicionalmente estável, totalmente explícito, e esparso de malha simplicial é obtido utilizando uma aproximação esparsa da inversa da matriz de Hodge. Nesta abordagem, a inversa da Matriz de Hodge deve ser explicitamente calculada para obter uma aproximação esparsa, e isso acarreta custos computacionais consideráveis.

Ainda em [5] é apresentado uma técnica de esparsificação para inversa da matriz de Hodge pelo particionamento recursivo da matriz em blocos. A ideia fundamental é aproximar as submatrizes por matrizes esparsas durante o processo de inversão por blocos. Experimentos numéricos mostram que o método leva a um menor número de operações no processo de inversão (ou seja, a um menor custo computacional) e é incondicionalmente estável.

Neste paper será mostrado que a utilização do algoritmo de Cuthill-McKee [2] sobre as matrizes de Hodge pode diminuir de uma maneira considerável o tempo de processamento para esparsificação recursiva.

\section{MATRIZES DE HODGE}

As Matrizes de Hodge são matrizes geradas a partir das relações constitutivas dos meios materiais. A essência destas matrizes está relacionada ao tipo de discretização, ou seja, serão construidas em função do tipo de malha utilizada. Se durante a discretização é utilizada uma malha estruturada como FDTD ${ }^{5}$, obtêm-se as matrizes de Yee-Hodge, por outro lado, se forem utilizadas malhas não estruturadas como no $\mathrm{FEM}^{6}$, têm-se as matrizes do tipo Galerkin-Hodge [6].

As matrizes de Yee-Hodge, geradas a partir de malhas hexaédricas, são diagonais. Com isso, o cálculo dos graus de liberdade das Equações de Maxwell, que envolve a inversão da matriz de Hodge, se torna algo trivial. Por outro lado, a discretização com elementos de Whitney gera a matrizes de Galerkin-Hodge, que são não diagonais, definida positiva, simétrica e esparsa. Essas matrizes possuem inversas tipicamente cheias, o que motiva o uso de técnicas para obtenção de uma matriz diagonal aproximada para a inversa dessa classe de matrizes. Em [5] encontramos o Método da Esparsificação. De maneira geral as Matrizes de Galerkin-Hodge podem ser calculadas pela expressão

$$
\mathbf{M}_{p}(\alpha)=\int_{\Omega} \alpha \omega_{p}^{i} \cdot \omega_{p}^{j} d \Omega
$$

onde $\alpha$ denota um dos campos escalares: $\epsilon$ (permissividade elétrica), $v$ (relutividade) ou $\sigma$ (condutividade elétrica do meio). $\omega_{p}^{i}$ e $\omega_{p}^{j}$ são 1 ou 2 -formas de Whitney e $\Omega$ é o domínio de integração.

A motivação para se obter explicitamente a inversa esparsa da matriz de Hodge é para calcular a solução do problema dado pelo sistema,

$$
\begin{gathered}
\partial_{t} \mathbf{b}+\mathbf{R e}=0 \\
-\partial_{t} \mathbf{M}_{1}(\epsilon) \mathbf{e}+\mathbf{R}^{T} \mathbf{M}_{2}(v) \mathbf{b}=0 .
\end{gathered}
$$

\footnotetext{
${ }^{4}$ Finite-Element Time-Domain.

${ }^{5}$ Finite-Difference Time-Domain.

${ }^{6}$ Finite Element Method.
} 
Esse sistema representa as Equações de Maxwell em sua forma discreta acrescida das relações constitutivas inseridas via as matrizes de Hodge $\mathbf{M}_{1}(\epsilon)$ e $\mathbf{M}_{2}(v)$ em cada passo de tempo. No sistema, temos que b e e são vetores grau de liberdade localizados nas faces e bordas da malha primal, $\mathbf{R}$ é a matriz de incidência face-aresta, $\mathbf{M}_{1}(\epsilon)$ e $\mathbf{M}_{2}(v)$ são matrizes de Hodge.

Em (2.2), b é naturalmente explícito. Para se obter um método explícito para o sistema é necessário resolver a segunda equação em (2.2) para e, e para isso é preciso calcular a inversa da matriz de Hodge $\mathbf{M}_{1}(\epsilon)$.

\section{O MÉTODO DA ESPARSIFICAÇÃO RECURSIVA}

O fato das Matrizes Yee-Hodge serem diagonais dispensa o uso do método da esparsificação recursiva, pois a inversa dessa classe de matrizes são facilmente obtidas sem grandes custos computacionais. Contudo, as Matrizes de Galerkin-Hodge são não diagonais, e por vezes, o cálculo de sua inversa envolve um custo computacional considerável. Sendo assim, o uso de técnicas como o Método da Esparsificação Recursiva torna-se aplicável a fim de diminuir esse custo computacional.

A técnica de Esparsificação Recursiva da inversa da matriz de Hodge consiste no particionamento recursivo desta matriz em blocos. A ideia fundamental é aproximar as submatrizes por matrizes esparsas durante o processo de inversão por blocos. Experimentos numéricos apresentados em [5] mostram que o método leva a um menor número de operações no processo de inversão (ou seja, a um menor custo computacional) e é incondicionalmente estável.

O método de esparsificação recursiva da inversa da matriz de Hodge é baseada na seguinte fórmula de inversão por bloco:

$$
\mathbf{D}_{i-1}^{-1}=\left[\begin{array}{cc}
\mathbf{Q}_{i}^{-1} & \mathbf{X}_{i} \\
\mathbf{X}_{i}^{T} & \mathbf{S}_{i}
\end{array}\right]
$$

onde $\mathbf{D}_{0}^{-1}=\mathbf{M}^{-1}$ e

$$
\begin{aligned}
\mathbf{Q}_{i} & =\mathbf{A}_{i}-\mathbf{B}_{i} \mathbf{D}_{i}^{-1} \mathbf{B}_{i}^{T} \\
\mathbf{X}_{i} & =-\mathbf{Q}_{i}^{-1} \mathbf{B}_{i} \mathbf{D}_{i}^{-1} \\
\mathbf{S}_{i} & =\mathbf{D}_{i}^{-1}-\mathbf{D}_{i}^{-1} \mathbf{B}_{i}^{T} \mathbf{X}_{i}
\end{aligned}
$$

As expressões em (3.1) e (3.2) surgem a partir do particionamento de uma matriz que é descrito da seguinte maneira:

$$
\mathbf{D}_{i-1}=\left[\begin{array}{cc}
\mathbf{A}_{\mathbf{i}} & \mathbf{B}_{\mathbf{i}} \\
\mathbf{B}_{\mathbf{i}}^{\mathbf{T}} & \mathbf{D}_{\mathbf{i}}
\end{array}\right] .
$$

Note que a inversão da matriz $\mathbf{D}_{0}$ de ordem $n \times n$ se reduz a inversão de uma matriz $\mathbf{D}_{1}$ de ordem $(n-q \times n-q)$ e uma matriz $\mathbf{Q}_{1}$ de ordem $(q \times q)$, onde $0<q<n$. A fórmula de inversão pode ser aplicada recursivamente na matriz $\mathbf{D}_{1}$, levando a uma sequência de tamanho decrescente de matrizes.

$$
\mathbf{M}=\mathbf{D}_{0}, \mathbf{D}_{1}, \mathbf{D}_{2}, \ldots, \mathbf{D}_{k}
$$


onde

$$
k=\left\lfloor\log _{q / n}(1 / n)\right\rfloor
$$

e $\mathbf{D}_{i}$ é o bloco de $\mathbf{D}_{i-1}$, para $i=1,2, \ldots, k$, tal que a inversa $\mathbf{M}^{-1}$ é obtida pelas substituições sucessivas através da sequência

$$
\mathbf{D}_{k}^{-1}, \mathbf{D}_{k-1}^{-1}, \ldots, \mathbf{D}_{1}^{-1}, \mathbf{D}_{0}^{-1}=\mathbf{M}^{-1}
$$

As matrizes em (3.6) podem ser aproximadas por matrizes esparsas, para se obter uma sequência de matrizes esparsas

$$
\mathbf{D}_{k_{s}}^{-1}, \mathbf{D}_{(k-1)_{s}}^{-1}, \ldots, \mathbf{D}_{0_{s}}^{-1}=\mathbf{M}_{s}^{-1}
$$

para aproximar a sequência descrita em (3.6).

Após cada inversão das matrizes em (3.6), a respectiva matriz $\mathbf{D}_{k}^{-1}$ é esparsificada para uma matriz $\mathbf{D}_{k_{s}}^{-1}$ de maneira que os coeficiente fora da diagonal da matriz $\mathbf{D}_{k}^{-1}=\left[d_{i j}\right]$ são zerados quando

$$
\left|d_{i \neq j}\right| \leq r \min \left|\operatorname{diag} \mathbf{D}_{k}^{-1}\right|
$$

onde $0<r<1$ é o parâmetro threshold.

Este critério é utilizado devido os maiores valores da matriz de Hodge estarem localizados em sua diagonal, induzindo desta forma naturalmente um valor de corte, que é dado por uma fração do menor coeficiente diagonal.

O interesse do método está em se obter de uma maneira eficiente uma aproximação para a inversa da matriz de Hodge que seja esparsa e que não perca as características da matriz inversa original, tais como a positividade definida e a forte localização dos elementos.

Em [5] é mostrado que o método não afeta a estabilidade do sistema apresentado em (2.2), devido ao fato do sinal dos autovalores não ser alterado e de se manter a forte concentração dos termos de maior grandeza em torno da diagonal principal.

\section{ALGORITMO DE CUTHILL-McKEE}

Dada uma matriz simétrica $\mathbf{M}=\left(m_{i, j}\right)$, de dimensões $n \times n$, define-se largura de banda como sendo a maior distância de um elemento não nulo à diagonal principal, que é dada por:

$$
\beta=\max |i-j|, \quad m_{i, j} \neq 0
$$

O algoritmo de Cuthill-Mckee trabalha com o objetivo de diminuir a largura de banda $(\beta)$ dessa matriz M, que é tratada pelo algoritmo como uma matriz de adjacência de um grafo.

A ideia básica do algoritmo é encontrar e efetuar uma permutação em $\mathbf{M}$ de forma que as entradas não nulas se aproximem da diagonal principal. Para encontrar tal permutação ele percorre todas as entradas da matriz verificando as não nulas e armazenado os valores $i$ e $j$ de sua posição 
numa matriz unidimensional que é gerada durante o processo, esses valores representam os nós de um grafo gerado pela matriz, que posteriormente será percorrido partindo sempre dos nós que possuem menos ligações, gerando assim a permutação que será aplicada em $\mathbf{M}$.

Na Figura 1 a seguir, note que o grafo é gerado a partir da matriz $\mathbf{M}$, a qual se deseja diminuir a largura de banda, a partir do grafo a permutação $q=\{4,1,5,2,3\}$ é construída e aplicada na matriz $\mathbf{M}$, gerando assim a matriz $\mathbf{M}^{\prime}$, onde já pode ser observado a alocação dos elementos não-nulos da matriz próximos à diagonal. Observe que a permutação $q=\{4,1,5,2,3\}$ é obtida partindo do número 4 que possui apenas uma ligação e seguindo a ordem crescente de ligações no grafo, atingindo assim todos os nós.

$$
\mathbf{M}=\left[\begin{array}{lllll}
1 & 0 & 0 & 4 & 1 \\
0 & 2 & 9 & 0 & 4 \\
0 & 1 & 3 & 0 & 7 \\
3 & 0 & 0 & 4 & 0 \\
2 & 8 & 6 & 0 & 5
\end{array}\right]
$$

a)

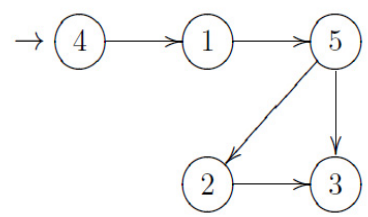

b)

$$
\mathbf{M}^{\prime}=\left[\begin{array}{lllll}
4 & 3 & 0 & 0 & 0 \\
4 & 1 & 1 & 0 & 0 \\
0 & 2 & 5 & 8 & 6 \\
0 & 0 & 4 & 2 & 9 \\
0 & 0 & 7 & 1 & 3
\end{array}\right]
$$

c)

Figura 1: (a) Matriz Simétrica M, (b) Grafo que gera a permutação $q=\{4,1,5,2,3\}$, (c) Matriz $\mathbf{M}^{\prime}$, que é obtida a partir da aplicação da permutação $q$ sobre a matriz $\mathbf{M}$.

Dessa forma o Algoritmo de Cuthill-Mckee faz com que os elementos não nulos de uma matriz fiquem concentrados próximo à diagonal principal, diminuindo a largura de banda da matriz e possibilitando obter ganhos computacionais quando utilizado juntamente com o método da esparsificação recursiva na inversão da Matriz de Hodge, como será visto posteriormente.

\section{APLICAÇÃO DO ALGORITMO DE CUTHILL-McKEE COM O MÉTODO DA ESPARSIFICAÇÃO RECURSIVA}

A aplicação do algoritmo de Cuthill-Mckee juntamente com o método da esparsificação recursiva tem como objetivo reduzir o tempo de processamento para a inversa da Matriz de Hodge, porém sem perder as características da matriz inversa original, como a positividade definida e a forte localização dos elementos não nulos próximo à diagonal.

Na Figura 1, pôde ser visto que o Algoritmo de Cuthill-Mckee aplica uma permutação na matriz M, e essa permutação faz com que os elementos não nulos de $\mathbf{M}$ se aproximem da diagonal principal, diminuindo assim a largura de banda da matriz. Na Figura 2 é mostrado uma Matriz de Hodge convencional e sua configuração após ser tratada pelo Algoritmo de Cuthill-Mckee.

Ao combinar o método da esparsificação recursiva com o algoritmo de Cuthill-Mckee pode ser observado que alguns blocos da matriz de Hodge será composta em grande parte por elementos nulos, esses blocos se referem a $\mathbf{B}_{i}$ e $\mathbf{B}_{i}^{T}$ apresentados em (3.3). Os elementos não nulos estarão concentrados na parte referente aos blocos $\mathbf{A}_{i}$ e $\mathbf{D}_{i}$, conforme separação em blocos apresentada em (3.3). 

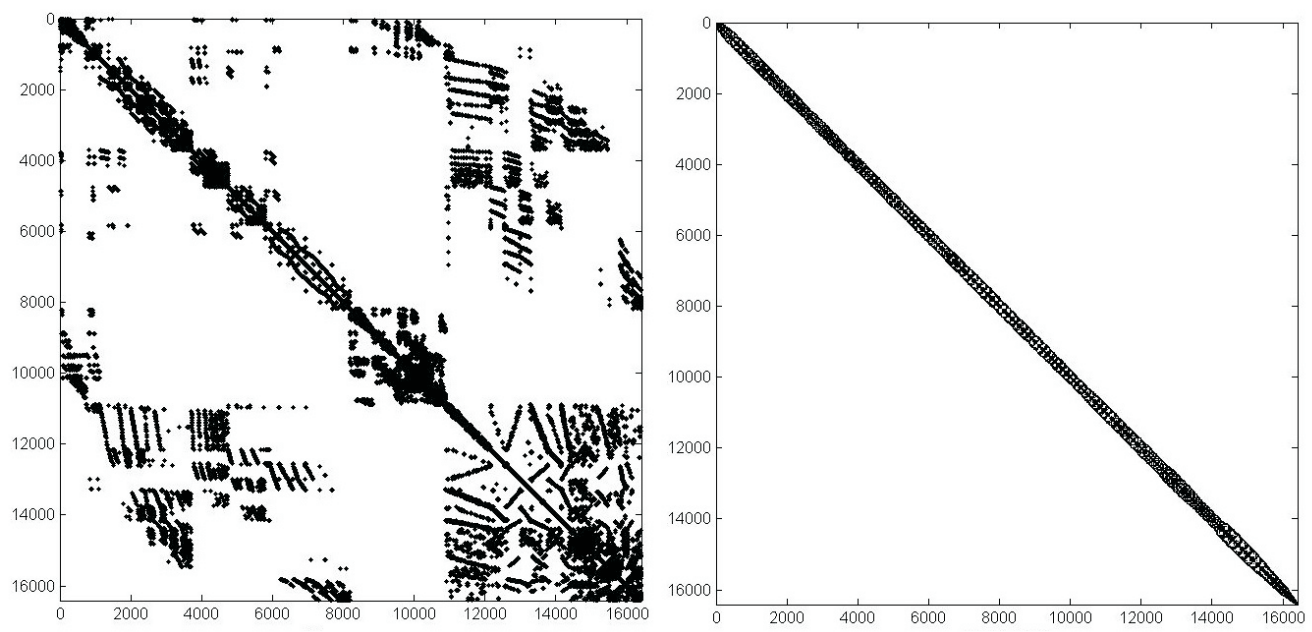

Figura 2: (a) Matriz de Hodge convencional. (b) Matriz de Hodge tratada pelo algoritmo de Cuthill-Mckee.

Essa nova configuração da matriz de Hodge proporciona uma diminuição considerável no tempo de processamento para obtenção de sua inversa esparsa através da utilização da esparsificação recursiva. A seguir apresentamos alguns resultados numéricos que evidenciam essa observação.

\section{RESULTADOS}

O objetivo desta seção é expor os resultados obtidos a partir da utilização da esparsificação recursiva juntamente com o algoritmo de Cuthill-Mckee na inversão das Matrizes de Hodge. Nela, compara-se o tempo de processamento computacional na aplicação da diagonalização de Cuthill-Mckee com o método da Esparsificação Recursiva e é feita uma simulação de guia de onda retangular 2D.

\subsection{Guia de onda retangular 2D}

Considere o guia de onda retangular, cujas paredes na direção $z$ são condutoras eletricamente perfeitas (PEC - perfect electric conductor) e cujo material interno é o ar.

O esquema Leap-frog [4] em termos de e e b para este problema será dado por

$$
\begin{aligned}
\mathbf{b}^{k+1} & =\mathbf{b}^{k}-\delta t \mathbf{G e}^{k-1 / 2} \\
\mathbf{e}^{k+1 / 2} & =\mathbf{e}^{k-1 / 2}+\delta t\left[\mathbf{M}_{0}^{-1}(\epsilon) \mathbf{G}^{T} \mathbf{M}_{1}(\nu) \mathbf{b}^{k}\right]
\end{aligned}
$$

onde $\mathbf{G}$ é a matriz de incidência nó-aresta e $\mathbf{M}_{0}(\epsilon)$ e $\mathbf{M}_{1}(v)$ são as as matrizes de GalerkinHodge [5].

A Figura 3 apresenta a distribuição do campo elétrico na região central do guia $(x=a / 2)$ com 17.400 passos de tempo, em contraste com a solução analítica. Observe que a solução do método 
com formas diferenciais é concordante com a solução analítica e, o campo é completamente absorvido pela camada PML.

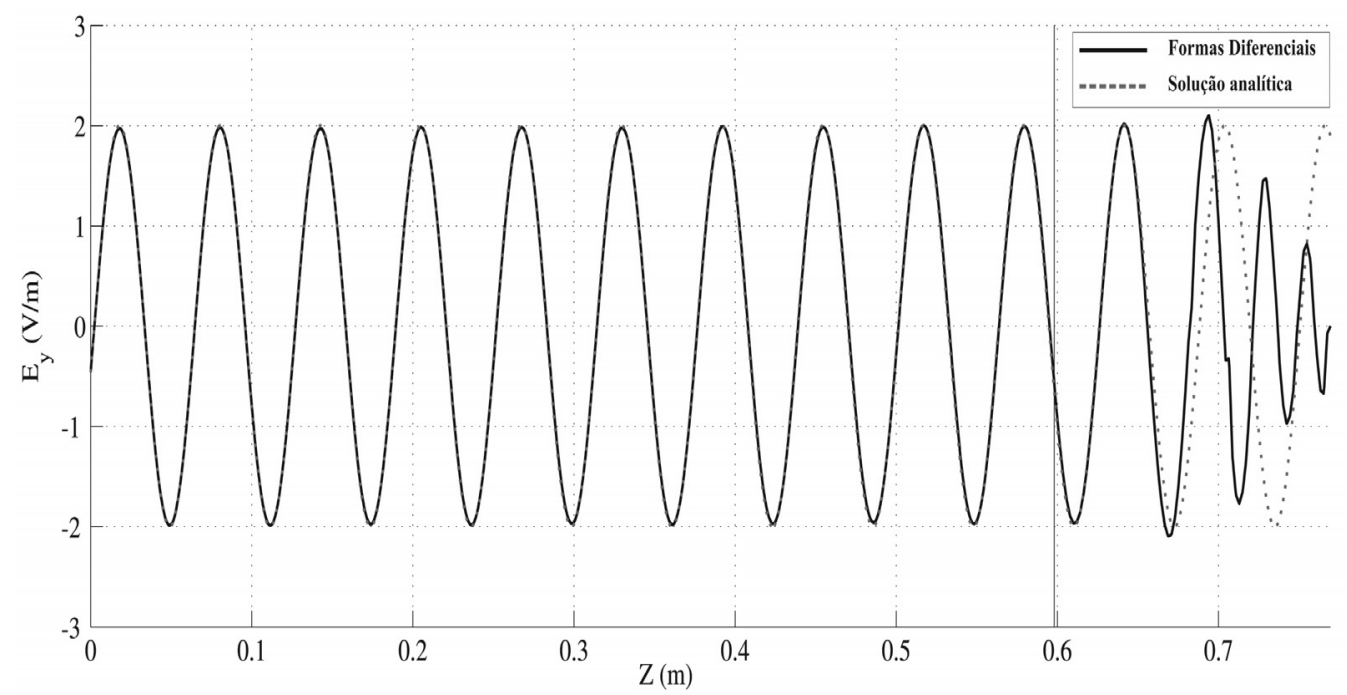

Figura 3: Campo elétrico $\mathcal{E}_{y}$ no meio do guia $x=a / 2$ com 17.400 passos de tempo.

\subsection{Tempo de Processamento}

Para se mostrar a redução do tempo de processamento devido a aplicação da diagonalização de Cuthill-McKee, é fixado o parâmetro threshold em $r=1 \times 10^{-5}$ e comparamos o tempo de processamento do método da esparsificação recursiva com e sem a aplicação da diagonalização da matriz, para matrizes de Hodge de ordem $n \times n, \operatorname{com} n$ variando entre 50.000 a 75.000 .

Tabela 1: Tempo de Computação: Cuthill-Mckee $(\mathrm{CM}) \times$ Esparsificação Recursiva(ER).

\begin{tabular}{|c|c|c|c|}
\hline $\begin{array}{c}\text { Tamanho da matriz } \\
n\end{array}$ & $\begin{array}{c}\text { CM } \\
(\mathrm{s})\end{array}$ & $\begin{array}{c}\text { ER } \\
(\mathrm{s})\end{array}$ & $\begin{array}{c}\text { Speedup } \\
\text { CM/ER }\end{array}$ \\
\hline 50.000 & 17,58 & 28,85 & 0,6094 \\
55.000 & 21,70 & 46,48 & 0,4667 \\
60.000 & 29,27 & 76,99 & 0,3815 \\
65.000 & 42,42 & 422,87 & 0,1003 \\
70.000 & 396,41 & 1002,4 & 0,3955 \\
75.000 & 853,08 & 1513,9 & 0,5635 \\
\hline
\end{tabular}

A Tabela 1 mostra os resultados obtidos neste experimento, percebe-se uma redução significativa no tempo de processamento pela aplicação da diagonalização. Destacamos que para uma matriz de ordem $65.000 \times 65.000$ obtivemos um redução de $90 \%$ no tempo de processamento. 


\title{
7 CONCLUSÃO
}

Neste trabalho pôde ser observado uma redução expressiva no tempo de processamento para o método da Esparsificação Recursiva com o uso do algoritmo de Cuthill-Mckee. Este fato ocorre em decorrência da geração de blocos quase-nulos após a diagonalização da matriz proporcionando desta forma uma redução de operações a serem efetuadas.

\begin{abstract}
The solution of sparse linear systems of high order is inserted in various branches of science, such as engineering. Consequently, there has been a great effort to resolve or provide approximate solutions of such systems efficiently. This work combines the method of recursive sparsification with the Cuthill-McKee algorithm for a sparse approximation to the inverse of a class of sparse matrices called Hodge's Matrices.
\end{abstract}

Keywords: recursive Sparsification, Hodge's matrices, Cuthill-McKee algorithm.

\section{REFERÊNCIAS}

[1] A. Bossavit. "Computational Electromagnetism: Variational Formulation, Complementarity, Edge Elements", Academic Press, San Diego (1994).

[2] A. George \& J.W.H Liu. "Computer Solution of Large Sparse Positive Definite Systems", PrenticeHall (1981).

[3] B. He \& F.L. Teixeira. Geometric finite element discretization of Maxwell equations in primal and dual spaces. Physics Letters A, 349 (2006), 1-14, Elsevier.

[4] J. Keranen, J. Kangas, A. Ahola \& L. Kettunen. Implicit Yee-like scheme on tetrahedral mesh. Magnetics, IEEE Transactions, 32(2) (2002), 717-720.

[5] A.S. Moura, R.R. Saldanha, E.J. Silva, A.C. Lisboa, W.G. Facco \& N.Z. Lima. A recursive sparsification of the inverse hodge matrix. Magnetics, IEEE Transactions, 48 (2012), 611-614.

[6] A.S. Moura, R.R. Saldanha, E.J. Silva, A.C. Lisboa \& W.G. Facco. Discretization of the CFS-PML for computational electromagnetics using discrete differential forms. Microwave and Optical Technology Letters, 55(2) (2013), 351-357.

[7] A. Quaterorni, R. Sacco \& F. Saleri. Numerical Mathematics. Texts in Applied Mathematics, 37 (2007), Springer Berlin Heidelberg. 